\title{
On Identifiability of Object-Oriented Models
}

\author{
Markus Gerdin, Torkel Glad \\ Division of Automatic Control \\ Department of Electrical Engineering \\ Linköpings universitet, SE-581 83 Linköping, Sweden \\ WWW: http://www.control.isy.liu.se \\ E-mail: gerdin@isy.liu.se, torkel@isy.liu.se
}

5th December 2005

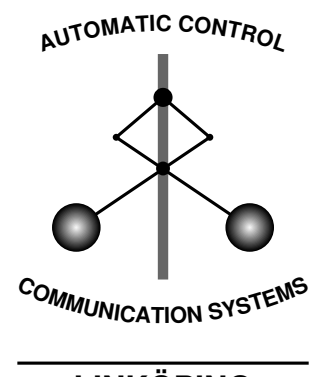

Report no.: LiTH-ISY-R-2710

Submitted to 14th IFAC Symposium on System Identification, SYSID-2006

Technical reports from the Control \& Communication group in Linköping are available at http://www.control.isy.liu.se/publications. 


\begin{abstract}
When estimating unknown parameters, it is important that the model is identifiable so that the parameters can be estimated uniquely. For nonlinear differentialalgebraic equation models with polynomial equations, a differential algebra approach to examine identifiability is available. This approach can be slow, so the present paper describes how this method can be modularized for objectoriented models. A characteristic set of equations is computed for components in model libraries, and stored together with the components. When an objectoriented model is built using such models, identifiability can be examined using the stored equations.
\end{abstract}

Keywords: Identifiability, Nonlinear systems, Algebraic methods, Object oriented modelling, Modelling, Identification, Descriptor systems 


\title{
ON IDENTIFIABILITY OF OBJECT-ORIENTED MODELS ${ }^{1}$
}

\author{
Markus Gerdin \& Torkel Glad
}

\author{
(gerdin, torkel)@isy. liu.se \\ Division of Automatic Control, Department of Electrical \\ Engineering, Linköping University, SE-581 83 Linköping, \\ Sweden
}

\begin{abstract}
When estimating unknown parameters, it is important that the model is identifiable so that the parameters can be estimated uniquely. For nonlinear differential-algebraic equation models with polynomial equations, a differential algebra approach to examine identifiability is available. This approach can be slow, so the present paper describes how this method can be modularized for objectoriented models. A characteristic set of equations is computed for components in model libraries, and stored together with the components. When an objectoriented model is built using such models, identifiability can be examined using the stored equations.
\end{abstract}

Keywords: Identifiability, Nonlinear systems, Algebraic methods, Object oriented modelling, Modelling, Identification, Descriptor systems

\section{INTRODUCTION}

A model structure is identifiable if it is possible to estimate its unknown parameters uniquely from measured input and output data. One important reason to examine identifiability of a model structure might be that the parameters represent physical properties that are of interest. (If that is not the case it might be sufficient to estimate certain combinations of the parameters such as sums, products or quotients.) Another reason could be that numerical search methods have difficulties in computing the parameters if the model structure is not identifiable.

Identifiability has been studied by many authors, e.g., Bellman and Åström (1970) and Walter (1982). Ljung (1999) is a standard reference in

\footnotetext{
1 This work has been supported by the Swedish Foundation for Strategic Research (SSF) through VISIMOD and EXCEL and by the Swedish Research Council (VR) which is gratefully acknowledged.
}

system identification, including identifiability. In (Ljung and Glad, 1994), a general method for examining identifiability in linear and nonlinear systems, both state-space systems and differentialalgebraic equations (DAE), is presented. However, this method uses differential algebra which suffers from high computational complexity, and can therefore only handle quite small systems. This contribution discusses how the modularized structure in object-oriented models can be used to speed up the computations. Modern modeling tools such as Modelica are based on objectoriented modeling, so this approach can be useful for models created using such tools.

\section{PRELIMINARIES}

In this section some preliminaries on objectoriented modeling and identifiability are presented. 


\subsection{Identifiability}

Identifiability of a model structure basically means that if two parameter values $\theta_{1}$ and $\theta_{2}$ give the same predictor, then $\theta_{1}=\theta_{2}$. This property can be local or global. Identifiability of a model structure actually only means that it is in principle possible to estimate unknown parameters uniquely. It does not guarantee that identification experiments give good results since the results, among other things, also depend on input signals and measurement accuracy. Nevertheless, it of course important that a model structure is identifiable if parameters are to be estimated.

As is common when examining identifiability for nonlinear systems, noise-free models will be treated in this contribution, so here the predictor will be the simulated output. For a discussion on this see, e.g., Ljung and Glad (1994) from which the basic setting that will be used is taken. This means that the model is specified by

$$
h_{i}(l(t), w(t), \theta(t), p)=0 \quad i=1,2, \ldots, r .
$$

Here $w(t) \in \mathcal{R}^{n_{w}}$ is a vector of measured input and output signals, $l(t) \in \mathcal{R}^{n_{l}}$ is a vector of internal variables, $\theta \in \mathcal{R}^{n_{\theta}}$ is a vector of unknown parameters, and $h_{i}(\cdot) \in \mathcal{R}$ while $p$ is the differentiation operator with respect to time, $p$. $x(t)=\frac{d x(t)}{d t}$.

In the paper by Ljung and Glad (1994), $w(t)$ is partitioned into inputs and outputs, but this is not necessary for our purposes.

The following result from Ljung and Glad (1994) will be used: Assume that a model structure is specified by (1) where the equations are polynomials and that the unknown parameters are timeinvariant, i.e. the equations

$$
\dot{\theta}(t)=0
$$

are included among the equations (1). Using Ritt's algorithm from differential algebra, (Ritt, 1966; Glad and Ljung, 1998), it is possible to compute a new set of equations of the form

$$
\begin{gathered}
A_{1}(w, p), \ldots, A_{n_{A}}(w, p), B_{1}\left(w, \theta_{1}, p\right), \\
B_{2}\left(w, \theta_{1}, \theta_{2}, p\right), \ldots, B_{n_{\theta}}\left(w, \theta_{1}, \theta_{2}, \ldots, \theta_{n_{\theta}}, p\right), \\
C_{1}(w, \theta, l, p), \ldots, C_{n_{l}}(w, \theta, l, p) .
\end{gathered}
$$

Typically it is possible to prove that the sets of equations (1) and (3) are equivalent, provided some conditions of the form

$$
s_{i}(l(t), w(t), \theta(t), p) \neq 0 \quad i=1,2, \ldots, n_{s}
$$

are satisfied. Identifiability is determined by the polynomials $B_{i}$ in (3). If the variables $\theta_{1}, \theta_{2}, .$. all occur exclusively in undifferentiated form in the $B_{i}$, then these polynomials give a triangular set of nonlinear equations for determining the $\theta_{i}$. If the $B_{i}$ have the form

$$
B_{i}=P_{i}(w, p) \theta_{i}-Q_{i}(w, p),
$$

i.e., a linear regression, then there is global identifiability, provided $P_{i}(w, p) \neq 0$.

While the result above, which is based on differential algebra, gives definite answers on identifiability for a wide class of systems, the computational complexity is high. Therefore this paper discusses how the modularized structure of object-oriented models can be used to reduce the computational complexity.

\subsection{Object-Oriented Modeling}

A central idea in object-oriented modeling is to build models by connecting submodels that represent physical parts of the system. For example when modeling an electrical circuit, the submodels can be components like voltage sources and resistors. These submodels are often standardized modules from model libraries. The modeling is usually performed through a graphical user interface where the submodels are connected. The most common language for object-oriented modeling is Modelica (Fritzson, 2004; Tiller, 2001).

In object-oriented modeling a complete model thus consists of a number of components, with equations describing them, and a number of equations describing the connections between the components. The complete model is therefore a system of differential-algebraic equations (DAE). Since the components represent different physical parts of the system, it is natural that they have independent parameters so that will be assumed in the present paper. For a model with $m$ components, the equations describing the components are written as

$$
f_{i}\left(l_{i}(t), w_{i}(t), \theta_{i}, p\right)=0 \quad i=1, \ldots, m .
$$

Here, $l_{i}(t) \in \mathcal{R}^{n_{l_{i}}}$ are internal variables, $w_{i}(t) \in$ $\mathcal{R}^{n_{w_{i}}}$ external variables that are used in the connections and $\theta_{i} \in \mathcal{R}^{\theta_{i}}$ unknown parameters, all in component $i$. As before $p$ is the differentiation operator with respect to time, $p \cdot x(t)=\frac{d x(t)}{d t}$. With $f_{i}(\cdot) \in \mathcal{R}^{n_{f_{i}}}$, it is assumed that $n_{f_{i}} \geq n_{l_{i}}$ so that there are at least as many equations as internal variables for each component. The equations describing the connections are written

$$
g(t, w(t))=0
$$

where

$$
w(t)=\left(\begin{array}{c}
w_{1}(t) \\
\vdots \\
w_{m}(t)
\end{array}\right) .
$$

The connection equations are typically simple equations like $a=b$ or $a+b+c=0$, but the framework also allows more complex connections. There are also typically equations of the form $w_{i}(t)=y(t)$, where $y$ is an external signal. To 
keep notation simple we assume that all external functions are represented by the time-variability of the function $g(\cdot)$. The dimension of $g(\cdot)$ is $n_{g}$.

To summarize, a complete object oriented model consists of the equations for the components and for the connections,

$$
\begin{aligned}
f_{i}\left(l_{i}(t), w_{i}(t), \theta_{i}, p\right) & =0 \quad i=1, \ldots, m . \\
g(t, w(t)) & =0 .
\end{aligned}
$$

This model can be analyzed using the method described in Section 2.1. Our main idea is however to separate the identifiability analysis into two stages. The first stage is to rewrite the model for a single component using the technique given by (3). We thus assume that the model

$$
f_{i}\left(l_{i}(t), w_{i}(t), \theta_{i}, p\right)=0
$$

can be rewritten in the equivalent form

$$
\begin{gathered}
A_{i, 1}\left(w_{i}, p\right), \ldots, A_{i, n_{A_{i}}}\left(w_{i}, p\right), \\
B_{i, 1}\left(w_{i}, \theta_{i, 1}, p\right), B_{i, 2}\left(w, \theta_{i, 1}, \theta_{i, 2}, p\right), \\
B_{i, n_{\theta_{i}}}\left(w_{i}, \theta_{i, 1}, \theta_{i, 2}, \ldots, \theta_{i, n_{\theta_{i}}}, p\right), \\
C_{i, 1}\left(w_{i}, \theta_{i}, l_{i}, p\right), \ldots, C_{i, n_{l_{i}}}\left(w_{i}, \theta_{i}, l_{i}, p\right) .
\end{gathered}
$$

An important part of the model for the analysis below is the set of $A_{i, j}$. These relations between the connecting variables are independent of the choice of the parameters.

Example 1. Consider a capacitor described by the voltage drop $w_{1}$, current $w_{2}$ and capacitance $\theta_{1}$. It is then described by (10) with

$$
f_{1}=\left(\begin{array}{c}
\theta_{1} \dot{w}_{1}-w_{2} \\
\dot{\theta}_{1}
\end{array}\right)
$$

If we consider only situations where $\dot{w}_{1} \neq 0$ we get the following series of equivalences.

$$
\begin{array}{cc}
\theta_{1} \dot{w}_{1}-w_{2}=0, & \dot{\theta}_{1}=0, \quad \dot{w}_{1} \neq 0 \\
\Leftrightarrow & \Leftrightarrow \\
\theta_{1} \dot{w}_{1}-w_{2}=0, & \theta_{1} \ddot{w}_{1}-\dot{w}_{2}=0, \quad \dot{w}_{1} \neq 0 \\
\Leftrightarrow & \Leftrightarrow \\
\theta_{1} \dot{w}_{1}-w_{2}=0, \quad \theta_{1} \dot{w}_{1} \ddot{w}_{1}-\dot{w}_{1} \dot{w}_{2}=0, \quad \dot{w}_{1} \neq 0 \\
\Leftrightarrow \\
\theta_{1} \dot{w}_{1}-w_{2}=0, \quad w_{2} \ddot{w}_{1}-\dot{w}_{1} \dot{w}_{2}=0, \quad \dot{w}_{1} \neq 0
\end{array}
$$

With the notation (11) we thus have

$$
\begin{aligned}
& A_{1,1}=w_{2} \ddot{w}_{1}-\dot{w}_{1} \dot{w}_{2} \\
& B_{1,1}=\theta_{1} \dot{w}_{1}-w_{2}
\end{aligned}
$$

and the function $s_{1}$ of $(4)$ is $\dot{w}_{1}$.

Example 2. Next consider an inductor where $w_{2}$ is the current, $w_{1}$ the voltage and $\theta_{1}$ the inductance. It is described by

$$
\theta_{1} \dot{w}_{2}=w_{1}, \quad \dot{\theta}_{1}=0
$$

Calculations similar to those of the previous example show that this is equivalent to

$$
\theta_{1} \dot{w}_{2}=w_{1}, \quad \ddot{w}_{2} w_{1}=\dot{w}_{2} \dot{w}_{1}
$$

provided $\dot{w}_{2} \neq 0$.

As discussed earlier, the transformation to (11) can always be performed for polynomial DAE. To show that calculations of this type in some cases also can be done for non-polynomial models, we consider a nonlinear resistor where the voltage drop is given by an arbitrary function.

Example 3. Consider a nonlinear resistor with the equation

$$
w_{1}=R\left(w_{2}, \theta_{1}\right)
$$

where it is assumed that the parameter $\theta_{1}$ can be uniquely solved from (17) if the voltage $w_{1}$ and the current $w_{2}$ are known, so that

$$
\theta_{1}=\phi\left(w_{1}, w_{2}\right) \text {. }
$$

Differentiating (17) once with respect to time and inserting (18) gives

$$
\dot{w}_{1}=R_{w_{2}}\left(w_{2}, \phi\left(w_{1}, w_{2}\right)\right) \dot{w}_{2}
$$

which is a relation between the external variables $w_{1}$ and $w_{2}$. We use the notation $R_{x}$ for the partial derivative of $R$ with respect to the variables $x$. In the special case with a linear resistor, where $R=\theta_{1} \cdot w_{2}$, this reduces to

$$
\begin{aligned}
\dot{w}_{1} & =\frac{w_{1}}{w_{2}} \dot{w}_{2} \\
\Leftrightarrow w_{2} \dot{w}_{1} & =w_{1} \dot{w}_{2}
\end{aligned}
$$

(assuming $w_{2} \neq 0$ ).

\section{MAIN RESULTS}

The main results of this paper concern how the modularized structure of object-oriented models can be used to examine identifiability in an efficient way.

Assume that all components are identifiable if the external variables $w_{i}$ of each component are measured. This means, that given measurements of

$$
w_{i} \quad i=1, \ldots, m
$$

the unknown parameters $\theta$ can be computed uniquely from the $B$ polynomials. When examining identifiability of the connected system it is not a big restriction to assume that the individual components are identifiable since information is removed when not all $w_{i}$ are measured. (Remember that all components have unique parameters.)

When the components have been connected, the only knowledge available about the $w_{i}$ is the $A$ polynomials and the equation $g(t, w(t))=$ 
0. (Remember that any known external signals are included in the time-variability of $g$.) The connected system is thus identifiable if the $w_{i}$ can be computed from

$$
\begin{gathered}
A_{i j}\left(w_{i}(t), p\right)=0 \quad\left\{\begin{array}{l}
i=1, \ldots, m \\
j=1, \ldots, n_{A_{i}}
\end{array}\right. \\
g(t, w(t))=0 .
\end{gathered}
$$

Note that this means that all $w(t)$ are algebraic variables (not differential), so that no initial conditions can be specified for any component of $w(t)$. If, on the other hand, there are several solutions to the equations (22) then these different solutions can be inserted into the $B$ polynomials, so there are also several possible parameter values. In this case the connected system is therefore not identifiable. Note again that measured inputs and outputs lead to equations of the form $w_{i}(t)=u(t)$, where the function $u$ is included in the timevariability of $g$.

The result is formalized in the following theorems. Note that the distinction between global and local identifiability was not discussed above, but this will be done below.

\subsection{Global Identifiability}

Global identifiability means that there is a unique solution to the identification problem, given that the measurements are informative enough. For a subsystem (10) that can be rewritten in the form (11) global identifiability means that the $B_{i, j}$ can be solved uniquely to give the $\theta_{i, j}$. In other words there exist functions $\psi$, that can in principle be calculated from the $B_{i, j}$, such that

$$
\theta_{i}=\psi_{i}\left(w_{i}, p\right)
$$

We then have the following formal result on identifiability.

Theorem 4. Consider an object-oriented model where the components are globally identifiable and thus can be described in the form (23). A sufficient condition for the total model to be globally identifiable is that (22) can be solved uniquely for the $w_{i}$. If all the functions $\psi_{i}$ of (23) are injective then this condition is also necessary.

Proof. If (22) gives a global solution for $w(t)$, then this solution can be inserted into the $B$ polynomials to give a global solution for $\theta$ since the components are globally identifiable. The connected system is thus globally identifiable. If there are several solutions for $w_{i}$ and the functions $\psi_{i}$ of (23) are injective, then there are also several solutions for $\theta$, so the system is not globally identifiable since the identification problem has more than one solution.

\subsection{Local Identifiability}

Local identifiability of a model structure means that locally there is a unique solutions to the identification problem, but globally there may be more than one solution. This means that the description (23) is valid only locally. We get the following result on local identifiability.

Theorem 5. Consider an object-oriented model where the components are locally identifiable and thus can be locally described in the form (23). A sufficient condition for the total model to be locally identifiable is that $(22)$ can be solved locally uniquely for the $w_{i}$. If all the functions $\psi_{i}$ of (23) are locally injective then this condition is also necessary.

Proof. If (22) gives a locally unique solution for $w(t)$, then this solution can be inserted into the $B$ polynomials to give a local solution for $\theta$ since the components are locally identifiable. The connected system is thus locally identifiable. If there locally are several solutions for $w_{i}$ and the functions $\psi_{i}$ of (23) are injective, then there are also several local solutions for $\theta$, so the system is not locally identifiable since the identification problem locally has more than one solution.

\section{APPLYING THE RESULTS}

The techniques discussed above are intended to be used when examining identifiability for objectoriented models. Since each component must be transformed into the form

$$
\begin{gathered}
A_{i, 1}\left(w_{i}, p\right), \ldots, A_{i, n_{A_{i}}}\left(w_{i}, p\right), \\
B_{i, 1}\left(w_{i}, \theta_{i, 1}, p\right), B_{i, 2}\left(w, \theta_{i, 1}, \theta_{i, 2}, p\right), \\
\quad B_{i, n_{\theta_{i}}}\left(w_{i}, \theta_{i, 1}, \theta_{i, 2}, \ldots, \theta_{i, n_{\theta_{i}}}, p\right), \\
C_{i, 1}\left(w_{i}, \theta_{i}, l_{i}, p\right), \ldots, C_{i, n_{l_{i}}}\left(w_{i}, \theta_{i}, l_{i}, p\right),
\end{gathered}
$$

the first step is to perform these transformations using, e.g., differential algebra (Ljung and Glad, 1994). The transformed version of the components can then be stored together with the original model equations in model libraries. As the transformation is calculated once and for all, it should also be possible to use other methods than differential algebra to make the transformation into the form (24). As mentioned above, this could make it possible to handle systems described by non-polynomial differential-algebraic equations.

When an object-oriented model has been composed of components for which the transformation into the form (24) is known, identifiability of the complete model,

$$
\begin{aligned}
f_{i}\left(l_{i}(t), w_{i}(t), \theta_{i}, p\right) & =0 \quad i=1, \ldots, m . \\
g(w(t)) & =0
\end{aligned}
$$


can be checked by examining the solutions of the differential-algebraic equation

$$
\begin{gathered}
A_{i j}\left(w_{i}(t), p\right)=0 \quad\left\{\begin{array}{l}
i=1, \ldots, m \\
j=1, \ldots, n_{A_{i}}
\end{array}\right. \\
g(w(t))=0 .
\end{gathered}
$$

The number of solutions to this differentialalgebraic equation then determines if the system is identifiable, as discussed in Theorem 4 and 5 . Note that the number of solutions could vary with $t$, so that the system is identifiable at some time instances and not at other.

The number of solutions of the differentialalgebraic equation (26) could be checked in different ways, and some are listed below.

\subsection{Differential Algebra}

If the system equations are polynomial, then one obvious way to check the number of solutions is to use differential algebra in a similar way as was done to achieve the form (24). This method can be slow in some cases, but it always gives definite answers. However, it some cases this approach should be faster than to derive the transformation to the form (24) for the complete object-oriented model.

Differential algebra can be used to examine both local and global identifiability, but requires that the equations are polynomial.

\subsection{Kunkel \& Mehrmann's Test}

Kunkel and Mehrmann (2001) describe a method to examine the properties of nonlinear differentialalgebraic equations through certain rank tests. Among other things, it is possible to determine the number of variables that are differential variables, and the number of variables that are algebraic variables. The algebraic variables can be calculated from the equations at each time instant, so (26) is locally uniquely solvable if all $w(t)$ are algebraic variables, so that no initial conditions can be specified.

\subsection{Manual Inspection}

For smaller models it may be possible to examine the solvability of (26) by inspection of the equations and manual calculations. This can of course not be developed into a general procedure, but may still be a good approach in some cases.

Manual inspection can be used to check both local and global identifiability.

\section{EXAMPLES}

In this section the techniques described in the paper are exemplified on a minimal model library consisting of a resistor model, an inductor model, and a capacitor model. Note that these components have corresponding components for example within mechanics and fluid systems (c.f., bond graphs). In this small example, all variables are external.

The transformation into the form (3) was performed in Section 2.2, so we shall here examine the identifiability of different connections of the components.

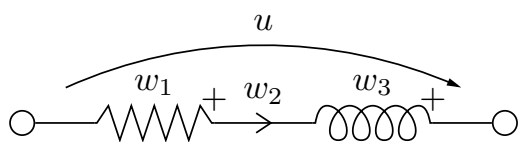

Fig. 1. A resistor and an inductor connected in series.

Example 6. Consider a nonlinear resistor and an inductor connected in series where the current $w_{2}=f$ and total voltage $u$ are measured as shown in Fig. 1. Denote the voltage over the resistor with $w_{1}$ and the voltage over the inductor with $w_{3}$. Using Examples 2 and 3 we get the equations

$$
\begin{aligned}
\dot{w}_{1} & =R_{w_{2}}\left(w_{2}, \phi\left(w_{1}, w_{2}\right)\right) \dot{w}_{2} \\
\ddot{w}_{2} w_{3} & =\dot{w}_{2} \dot{w}_{3}
\end{aligned}
$$

for the components and the equation

$$
w_{1}+w_{3}=u
$$

for the connections. Differentiating the last equation once gives

$$
\dot{w}_{1}+\dot{w}_{3}=\dot{u}
$$

The system of equations (27) (with $w_{1}, \dot{w}_{1}, w_{3}$, and $\dot{w}_{3}$ as unknowns) has the Jacobian

$$
\left(\begin{array}{cccc}
-R_{w_{2}, w_{1}} \dot{w}_{2} & 1 & 0 & 0 \\
0 & 0 & \ddot{w}_{2} & -\dot{w}_{2} \\
1 & 0 & 1 & 0 \\
0 & 1 & 0 & 1
\end{array}\right)
$$

where

$$
R_{w_{2}, w_{1}}=\frac{\partial}{\partial w_{1}}\left(R_{w_{2}}\left(w_{2}, \phi\left(w_{1}, w_{2}\right)\right)\right) .
$$

The Jacobian has the determinant $-R_{w_{2}, w_{1}} \cdot \dot{w}_{2}^{2}+$ $\ddot{w}_{2}$, so the system of equations is solvable for most values of the external variables. This means that the system is identifiable.

Example 7. Now consider two capacitors connected in series where the current $w_{2}=f$ and total voltage $u$ are measured as shown in Fig. 2 . Denote the voltages over the capacitors with $w_{1}$ 


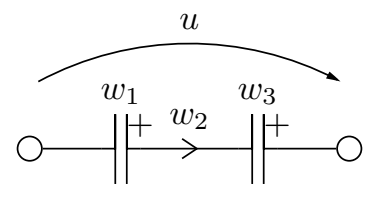

Fig. 2. Two capacitors connected in series.

and $w_{3}$ respectively. Using Example 1 we get the equations

$$
\begin{aligned}
& w_{2} \ddot{w}_{1}=\dot{w}_{1} \dot{w}_{2} \\
& w_{2} \ddot{w}_{3}=\dot{w}_{3} \dot{w}_{2}
\end{aligned}
$$

for the components. The connection is described by the equation

$$
w_{1}+w_{3}=u \text {. }
$$

These equations directly give that if

$$
\begin{aligned}
& w_{1}(t)=\phi_{1}(t) \\
& w_{3}(t)=\phi_{3}(t)
\end{aligned}
$$

is a solution, then so are all functions of the form

$$
\begin{aligned}
& w_{1}(t)=(1+\lambda) \phi_{1}(t) \\
& w_{3}(t)=\phi_{3}(t)-\lambda \phi_{1}(t)
\end{aligned}
$$

for scalar $\lambda$. Since (14b) implies that the capacitance is an injective function of the derivative of the voltage, this shows that the system is not identifiable.

\section{CONCLUSIONS}

This paper has shown how the structure of objectoriented models can be used to simplify examination of identifiability. For components in model libraries, the transformation to the form (24) is computed once and for all and stored with the component. This makes it possible to only consider a smaller number of equations when examining identifiability for an object-oriented model composed of such components. Although the method described in this paper may suffer from high computational complexity (depending, among other things, on the method selected for deciding the number of solutions for (26)), it can make the situation much better than when trying to use to use the differential-algebra approach described in (Ljung and Glad, 1994) on a complete model.

The technique could be included in tools for object-oriented modeling such as Dymola and Openmodelica. Preferably, this could be part of a complete set of system identification routines linked to the modeling software. The identification routines could either be included directly in the modeling software, or as external software that interacts with the modeling software.

Future work could include to examine if it is possible to make the method fully automatic, so that it can be included in modeling tools and to examine if other system analysis or design methods can benefit from the modularized structure in objectoriented models. It could also be interesting to examine the case when several components share the same parameter. This could occur for example if the different parts of the system are affected by environmental parameters such as temperature and fluid constants.

\section{REFERENCES}

Bellman, R. and K. J. Åström (1970). On structural identifiability. Mathematical Biosciences 7(3-4), 329-339.

Fritzson, P. (2004). Principles of Object-Oriented Modeling and Simulation with Modelica 2.1. Wiley-IEEE. New York.

Glad, S. T. and L. Ljung (1998). Identifiability with constraints. In: NOLCOS 1998. Enschede, the Netherlands. pp. 455-458.

Kunkel, P. and V. Mehrmann (2001). Analysis of over- and underdetermined nonlinear differential-algebraic systems with application to nonlinear control problems. Mathematics of Control, Signals, and Systems 14(3), 233-256.

Ljung, L. (1999). System Identification - Theory for the User. Information and System Sciences Series. Second ed. Prentice Hall PTR. Upper Saddle River, N.J.

Ljung, L. and T. Glad (1994). On global identifiability for arbitrary model parametrizations. Automatica 30(2), 265-276.

Ritt, J. F. (1966). Differential Algebra. Dover. New York.

Tiller, M. (2001). Introduction to Physical Modeling with Modelica. Kluwer. Boston, Mass.

Walter, E. (1982). Identifiability of State Space Models with Applications to Transformation Systems. Vol. 46 of Lecture Notes in Biomathematics. Springer-Verlag. Berlin, Heidelberg, New York. 


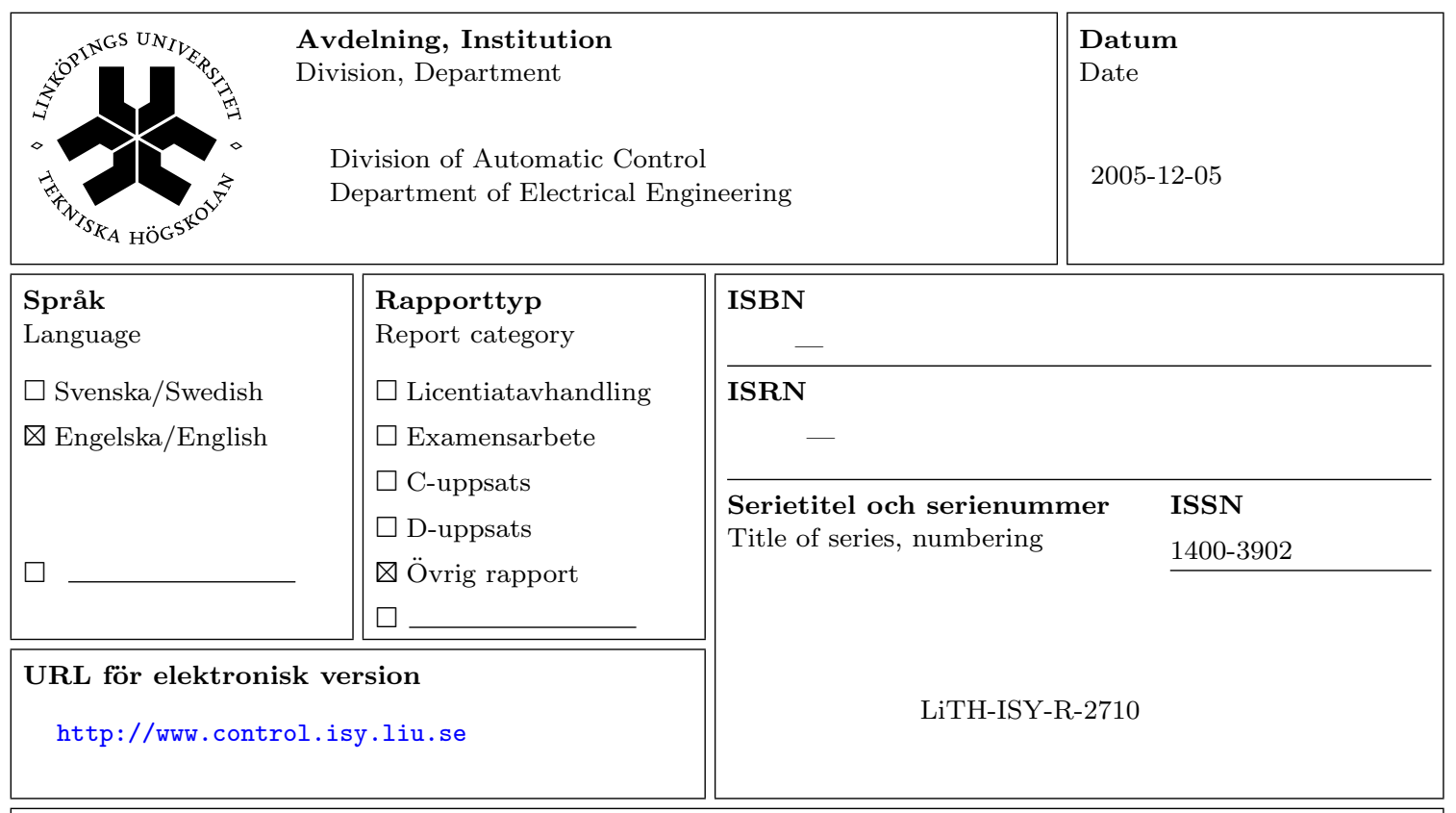

Titel On Identifiability of Object-Oriented Models

Title

Ints

Författare Markus Gerdin, Torkel Glad

Author

\section{Sammanfattning}

Abstract

When estimating unknown parameters, it is important that the model is identifiable so that the parameters can be estimated uniquely. For nonlinear differential-algebraic equation models with polynomial equations, a differential algebra approach to examine identifiability is available. This approach can be slow, so the present paper describes how this method can be modularized for object-oriented models. A characteristic set of equations is computed for components in model libraries, and stored together with the components. When an objectoriented model is built using such models, identifiability can be examined using the stored equations. 